\title{
A CONVERGENCE ANALYSIS OF STOCHASTIC COLLOCATION METHOD FOR NAVIER-STOKES EQUATIONS WITH RANDOM INPUT DATA
}

\author{
HOANG TRAN*, CATALIN TRENCHEA ${ }^{\dagger}$, AND CLAYTON WEBSTER ${ }^{\ddagger}$
}

\begin{abstract}
Stochastic collocation method has proved to be an efficient method and been widely applied to solve various partial differential equations with random input data, including NavierStokes equations. However, up to now, rigorous convergence analyses are limited to linear elliptic and parabolic equations; its performance for Navier-Stokes equations was demonstrated mostly by numerical experiments. In this paper, we provide an error analysis of stochastic collocation method for a semi-implicit Backward Euler discretization for NSE and prove the exponential decay of the interpolation error in the probability space. Our analysis indicates that due to the nonlinearity, as final time $T$ increases and NSE solvers pile up, the accuracy may be reduced significantly. Subsequently, the theoretical results are illustrated by the numerical test of time dependent fluid flow around a bluff body.
\end{abstract}

1. Introduction. Flow of liquids and gases is ubiquitous in nature and obtaining an accurate prediction of these flows is a central difficulty in diverse problems such as global change estimation, improving the energy effciency of engines, controlling dispersal of contaminants, designing biomedical devices and many other venues. Most applications of fluid flows in engineering and science are affected by uncertainty in the input data and mathematical models, e.g., forcing terms, wall roughness, material properties, source and interaction terms, geometry, model coefficients, etc. In this case, it is necessary to introduce uncertainty in mathematical models to assess the reliability of predictions based on numerical simulations.

The literature on numerical methods for stochastic differential equations has grown extensively in the last decade. The Monte Carlo sampling method is the classical and most popular approach for approximating expected values and other statistical moments of quantities of interest (QoI) based on the solution of PDEs with random inputs. While being very flexible and easy to implement, Monte Carlo method requires a very large number of samples to achieve small errors. Recently, other approaches have been proposed that often feature fast convergence. These include stochastic Galerkin methods, stochastic collocation methods, and perturbation, Neumann and Taylor expansion methods.

Stochastic collocation methods (SCM) have emerged to be a modern, efficient technique for quantifying uncertainty in physical applications [MHZ05, NT09, XH05]. One advantage of SCMs concerns the much faster convergence rates, which can yield accurate predictions of the uncertainty at a small fraction of the cost of a MonteCarlo simulation, while maintaining an ensemble-based, non-intrusive approach. The better convergence behavior of SCMs, however, requires analyticity of the solutions with respect to the random variables. In [BNT07], such property was established and the error estimates of SCMs were given for elliptic PDEs. These results have been extended to linear parabolic equations in [ZG12]. Often in nonlinear scientific and engineering problems, particularly in Navier-Stokes equations, complex solutions arise

\footnotetext{
${ }^{*}$ Computer Science and Mathematics Division, Oak Ridge National Laboratory, 1 Bethel Valley Road, P.O. Box 2008, Oak Ridge TN 37831-6164. email: tranha@ornl.gov.

${ }^{\dagger}$ Department of Mathematics, University of Pittsburgh, Pittsburgh, PA, 15260, USA. email: trenchea@pitt.edu. Partially supported by Air Force under grant FA9550-09-1-0058.

${ }^{\ddagger}$ Computer Science and Mathematics Division, Oak Ridge National Laboratory, 1 Bethel Valley Road, P.O. Box 2008, Oak Ridge TN 37831-6164. email: webstercg@ornl.gov.
} 
and their dependence on the random input data varies rapidly. For these cases, the smoothness of solutions in probability space has been less known. Consequently, the accuracy of SCMs (and their variants) has been demonstrated mostly by numerical experiments rather than by rigorous error analysis.

In this article, based on the procedure in [BNT07], we establish, for the first time, some analyticity results of solutions of a fully discrete approximation for stochastic Navier-Stokes equations. The scheme we analyze is backward Euler with constant extrapolation (BECE), space-time error estimates of which were obtained in [TT00]:

$$
\begin{aligned}
\frac{u^{n+1}-u^{n}}{\Delta t}-\nu \Delta u^{n+1}+u^{n} \cdot \nabla u^{n+1}+\nabla p^{n+1} & =f^{n+1}, \\
\nabla \cdot u^{n+1} & =0 .
\end{aligned}
$$

We show that at a fixed time $T$, the exponential decay of interpolation errors of SCMs does occur, just like the case of linear PDEs. However, the errors of numerical methods for NSE frequently exhibit sharp growths in $T$, e.g., exponential growth in time of the space-time error in deterministic NSE approximations [HR90, Lay08], and SCMs seem not to be an exception. We verify herein that type of growth for the interpolation errors of SCMs. Particularly, our analysis indicates that as $T$ increases, the radius of analyticity may shrink exponentially, resulting in a reduction in accuracy.

The outline of the paper is as follows. In Section 2, we introduce the mathematical problem and the notation used throughout the paper, as well as a brief description of the stochastic collocation methods. In Section 3, we derive the analyticity of the fully discrete solution of (BECE) and the converenge rates of SCMs for this scheme. A computational experiment of time dependent flow around a circular cylinder illustrating our numerical analysis is given in Section 4. Finally, concluding remarks are followed in Section 5.

1.1. Related works. Many variations have been introduced to improve the efficiency of stochastic numerical methods including sparse grid collocation [NTW08], anisotropic sparse grid collocation [NTW08b], and sparse polynomial bases [TS07]. The exponential decay of interpolation errors and the deterioration of accuracy in long time, which we analyze herein, have been indicated in the literature, e.g., [Luc04, WK06, WLSB08]. Not surprisingly, there have been many techniques developed to deal with stochastic nonlinear problems, whose solutions exhibit sharp variation and even discontinuity with respect to random data. These include domain decomposition of parametric spaces (multi-element polynomial chaos [WK06b], multi-element probabilistic collocation [FWK08]), wavelet expansions [MKNG04], [MNGK04], refinement strategies that focus on regions of irregular behavior (adaptively sparse-grid SCM [GWZ13], adaptive polynomial chaos [WK05]) and parameterization strategies for QoIs whose patterns are known [WLB07]. While these approaches clearly show significant improvements in efficiency and accuracy in various linear and nonlinear applications, their error estimates for nonlinear problems have not been provided. The discrete scheme (BECE) we study here is just one method in the class of multi-step backward differentiation methods coupled with semi-implicit or explicit scheme for the nonlinear terms, see [DFJ74, BDK82] among others. These schemes are attractive that each time step requires only one discrete Stokes system and linear solve. In addition, in many cases, no time step restriction is needed for stability and convergence. Space-time convergence estimates for (BECE) is derived in [TT00]. For numerical analysis for higher order methods, we refer to [GR79, HS07, Ing13]. The application of non-iterative extrapolating schemes in modeling engineering flows can be found 
in [SS02] (turbulent flows induced by wind turbine motion), [ACH06] (reacting flows in complex geometries such as gas turbine combustors), [MMOU06] (turbulent flows transporting particles).

\section{Problem setting.}

2.1. Stochastic incompressible Navier-Stokes equation. Let $D$ be a convex bounded polygonal domain in $\mathbb{R}^{m}(m=2,3)$ and let $(\Omega, \mathcal{F}, P)$ be a complete probability space. Here $\Omega$ is the set of outcomes, $\mathcal{F} \subset 2^{\Omega}$ is the $\sigma$-algebra of events, and $P: \mathcal{F} \rightarrow[0,1]$ is a probability measure. We define two random fields: the viscosity field $\nu(x, \omega): \bar{D} \times \Omega \rightarrow \mathbb{R}$ and the forcing field $f(t, x, \omega):[0, T] \times \bar{D} \times \Omega \rightarrow \mathbb{R}^{m}$.

The stochastic incompressible Navier-Stokes problem can be written as follows: find a random velocity, $u:[0, T] \times \bar{D} \times \Omega \rightarrow \mathbb{R}^{m}$ and random pressure $p:[0, T] \times \bar{D} \times$ $\Omega \rightarrow \mathbb{R}$, such that $P$-almost surely (a.s.) the following equation holds in $(0, T] \times D \times \Omega$ :

$$
\begin{gathered}
\partial_{t} u-\nu \Delta u+u \cdot \nabla u+\nabla p=f, \\
\nabla \cdot u=0,
\end{gathered}
$$

subject to the initial condition

$$
u(0, x, \omega)=u_{0}(x), \text { on } D \times \Omega,
$$

and the boundary condition

$$
u(t, x, \omega)=0, \quad \text { on }(0, T] \times \partial D \times \Omega .
$$

Let $\mathbf{y}=\left(y_{1}, \ldots, y_{d}\right)$ denote a $d$-dimensional random variable in $(\Omega, \mathcal{F}, P)$ and define the space $L_{P}^{2}(\Omega)$ comprising all random variables $\mathbf{y}$ satisfying

$$
\sum_{n=1}^{d} \int_{\Omega}\left|y_{n}(\omega)\right|^{2} d P(\omega)<\infty
$$

Then the following Hilbert spaces can be defined

$$
\begin{aligned}
& V=L^{2}\left(0, T ; H_{0}^{1}(D)\right) \otimes L_{P}^{2}(\Omega) \text { with norm }\|u\|_{V}^{2}=\int_{0}^{T} \int_{D} \mathbb{E}\left[|\nabla u|^{2}\right] d x d t, \\
& W=L^{2}\left(0, T ; L_{0}^{2}(D)\right) \otimes L_{P}^{2}(\Omega) \text { with norm }\|p\|_{W}^{2}=\int_{0}^{T} \int_{D} \mathbb{E}\left[|p|^{2}\right] d x d t .
\end{aligned}
$$

In order to define the weak form of the Navier-Stokes equations we introduce two continuous bilinear forms

$$
\begin{aligned}
& a(u, v)=2 \nu \sum_{i, j=1}^{m} \int_{D} D_{i j}(u) D_{i j}(v) d x, \quad \forall u, v \in H^{1}(D), \\
& b(v, q)=-\int_{D} q \nabla \cdot v d x, \quad \forall q \in L^{2}(D), v \in H^{1}(D),
\end{aligned}
$$

where $D_{i j}(v)=\frac{1}{2}\left(\partial v_{i} / \partial x_{j}+\partial v_{j} / \partial x_{i}\right)$, and the continuous trilinear form

$$
c(w ; u, v)=\frac{1}{2} \sum_{i, j=1}^{m}\left(\int_{D} w_{j}\left(\frac{\partial u_{i}}{\partial x_{j}}\right) v_{i} d x-\int_{D} w_{j}\left(\frac{\partial v_{i}}{\partial x_{j}}\right) u_{i} d x\right), \quad \forall w, u, v \in H^{1}(D)
$$


We now define weak solutions of the problem (2.1): a pair $(u, p) \in V \times W$ is a weak solution of (2.1) if it satisfies the initial condition $u(0, x, \omega)=u_{0}(x, \omega)$ and for $T>0$

$$
\begin{gathered}
\mathbb{E}\left[\left(\partial_{t} u, v\right)\right]+\mathbb{E}[a(u, v)]+\mathbb{E}[c(u ; u, v)]+\mathbb{E}[b(v, p)] \\
=\mathbb{E}[(f, v)], \text { for all } v \in H_{0}^{1}(D) \otimes L_{P}^{2}(\Omega), \\
\mathbb{E}[b(u, q)]=0, \text { for all } q \in L_{0}^{2}(D) \otimes L_{P}^{2}(\Omega) .
\end{gathered}
$$

2.2. Finite dimensional noise assumption. In many problems the source of randomness can be approximated using just a small number of uncorrelated or independent random variables; take, for example, the case of a truncated KarhunenLoève expansion, [Loe87]. This motivates us to make the following assumption.

ASSUMPTION 2.1. The random input functions of the equation (2.1) have the form

$$
\begin{aligned}
\nu(x, \omega) & =\nu(x, \mathbf{y}(\omega)), \text { on } \bar{D} \times \Omega, \\
f(t, x, \omega) & =f(t, x, \mathbf{y}(\omega)), \text { on }[0, T] \times \bar{D} \times \Omega,
\end{aligned}
$$

where $\mathbf{y}(\omega)=\left(y_{1}(\omega), \ldots, y_{d}(\omega)\right)$ is a vector of real-valued random variables with mean value zero and unit variance.

We will denote with $\Gamma_{n} \equiv y_{n}(\Omega)$ the image of $y_{n}, \Gamma=\prod_{n=1}^{d} \Gamma_{n}$ and assume that the random variables $\left[y_{1}, \ldots, y_{d}\right]$ have a joint probability density function $\rho$ : $\Gamma \rightarrow \mathbb{R}_{+}$, with $\rho \in L^{\infty}(\Gamma)$. Hence the probability space $(\Omega, \mathcal{F}, P)$ can be replaced by $\left(\Gamma, \mathcal{B}^{d}, \rho d \mathbf{y}\right)$, where $\mathcal{B}^{d}$ is the $d$-dimensional Borel space.

Similar to $V$ and $W$, we can define $V_{\rho}$ and $W_{\rho}$ as

$$
\begin{gathered}
V_{\rho}=L^{2}\left(0, T ; H_{0}^{1}(D)\right) \otimes L_{\rho}^{2}(\Gamma) \text { with norm }\|u\|_{V_{\rho}}^{2}=\int_{\Gamma}\|u\|_{L^{2}\left(0, T ; H_{0}^{1}(D)\right)}^{2} \rho d \mathbf{y}, \\
W_{\rho}=L^{2}\left(0, T ; L_{0}^{2}(D)\right) \otimes L_{\rho}^{2}(\Gamma) \text { with norm }\|p\|_{W_{\rho}}^{2}=\int_{\Gamma}\|p\|_{L^{2}\left(0, T ; L_{0}^{2}(D)\right)}^{2} \rho d \mathbf{y} .
\end{gathered}
$$

After making Assumption 2.1, the solution $(u, p)$ of the stochastic NSE (2.2) can be described by just a finite number of random variables, i.e., $u(\omega, x)=u\left(y_{1}(\omega), \ldots, y_{d}(\omega), x\right)$, $p(\omega, x)=p\left(y_{1}(\omega), \ldots, y_{d}(\omega), x\right)$. Thus, the goal is to approximate the function $u=$ $u(\mathbf{y}, x)$ and $p=p(\mathbf{y}, x)$, where $\mathbf{y} \in \Gamma$ and $x \in \bar{D}$. Observe that the stochastic variational formulation (2.2) has a "deterministic" equivalent which is the following: find $u \in V_{\rho}, p \in W_{\rho}$ satisfying the initial condition and, for $T>0$

$$
\begin{gathered}
\int_{\Gamma} \rho\left(\partial_{t} u, v\right) d \mathbf{y}+\int_{\Gamma} \rho a(u, v) d \mathbf{y}+\int_{\Gamma} \rho c(u ; u, v) d \mathbf{y}+\int_{\Gamma} \rho b(v, p) d \mathbf{y} \\
=\int_{\Gamma} \rho(f, v) d \mathbf{y}, \text { for all } v \in H_{0}^{1}(D) \otimes L_{\rho}^{2}(\Gamma), \\
\int_{\Gamma} \rho b(u, q) d \mathbf{y}=0, \text { for all } q \in L_{0}^{2}(D) \otimes L_{\rho}^{2}(\Gamma) .
\end{gathered}
$$

For a fixed $T$, the solution has the form $u(\omega, x)=u\left(y_{1}(\omega), \ldots, y_{d}(\omega), x\right), p(\omega, x)=$ $p\left(y_{1}(\omega), \ldots, y_{d}(\omega), x\right)$ and we use the notation $u(\mathbf{y}), p(\mathbf{y}), \nu(\mathbf{y}), f(\mathbf{y})$, and $u_{0}(\mathbf{y})$ in order to emphasize the dependence on the variable $\mathbf{y}$. Then, the weak formulation (2.3) for $T>0$ is equivalent to

$$
\begin{gathered}
\left(\partial_{t} u(\mathbf{y}), v\right)+a(u(\mathbf{y}), v)+c(u(\mathbf{y}) ; u(\mathbf{y}), v)+b(v, p(\mathbf{y})) \\
=(f(\mathbf{y}), v), \text { for all } v \in H_{0}^{1}(D), \rho \text {-a.e. in } \Gamma, \\
b(u(\mathbf{y}), q)=0, \text { for all } q \in L_{0}^{2}(D), \rho \text {-a.e. in } \Gamma .
\end{gathered}
$$


2.3. Collocation method. Denote conforming velocity, pressure finite element spaces based on an edge to edge triangulation of $D$ (with maximum triangle diameter h) by

$$
H_{h} \subset H_{0}^{1}(D), L_{h} \subset L_{0}^{2}(D) .
$$

We assume that $H_{h}$ and $L_{h}$ satisfy the usual discrete inf-sup condition. TaylorHood elements, discussed in [BS08], [G89], are one commonly used choice of velocitypressure finite element spaces. The discretely divergence free subspace of $H_{h}$ is

$$
V_{h}:=\left\{v_{h} \in H_{h}:\left(\nabla \cdot v_{h}, q_{h}\right)=0, \forall q_{h} \in L_{h}\right\} .
$$

The spatial discrete approximation of (2.4) can be written as: find $u_{h} \in L^{2}\left(0, T ; H_{h}\right) \otimes$ $L_{\rho}^{2}(\Gamma)$ and $p_{h} \in L^{2}\left(0, T ; L_{h}\right) \otimes L_{\rho}^{2}(\Gamma)$ satisfying initial condition and for $T>0$

$$
\begin{gathered}
\left(\partial_{t} u_{h}(\mathbf{y}), v_{h}\right)+a\left(u_{h}(\mathbf{y}), v_{h}\right)+c\left(u_{h}(\mathbf{y}) ; u_{h}(\mathbf{y}), v_{h}\right)+b\left(v_{h}, p_{h}(\mathbf{y})\right) \\
=\left(f(\mathbf{y}), v_{h}\right), \text { for all } v_{h} \in H_{h}, \rho \text {-a.e. in } \Gamma, \\
b\left(u_{h}(\mathbf{y}), q_{h}\right)=0, \text { for all } q_{h} \in L_{h}, \rho \text {-a.e. in } \Gamma .
\end{gathered}
$$

or more simply: find $u_{h} \in L^{2}\left(0, T ; V_{h}\right) \otimes L_{\rho}^{2}(\Gamma)$ satisfying initial condition and for $T>0$

$$
\begin{gathered}
\left(\partial_{t} u_{h}(\mathbf{y}), v_{h}\right)+a\left(u_{h}(\mathbf{y}), v_{h}\right)+c\left(u_{h}(\mathbf{y}) ; u_{h}(\mathbf{y}), v_{h}\right)=\left(f(\mathbf{y}), v_{h}\right), \\
\text { for all } v_{h} \in V_{h}, \rho \text {-a.e. in } \Gamma,
\end{gathered}
$$

We apply the stochastic collocation method to the weak form (2.5). Define $\mathcal{P}_{p}(\Gamma) \subset L_{\rho}^{2}(\Gamma)$ is the span of tensor product polynomials with degree at most $p=\left(p_{1}, \ldots, p_{d}\right)$. The dimension of $\mathcal{P}_{p}(\Gamma)$ is $N_{p}=\prod_{n=1}^{d}\left(p_{n}+1\right)$. We seek a numerical approximation to the solution of (2.5) in finite dimensional subspaces $V_{\rho, h}=$ $L^{2}\left(0, T ; H_{h}\right) \otimes \mathcal{P}_{p}(\Gamma)$ and $W_{\rho, h}=L^{2}\left(0, T ; L_{h}\right) \otimes \mathcal{P}_{p}(\Gamma)$.

The procedure for solving (2.5) is divided into two parts. First, for a fixed $T>$ 0 , at each collocation point (root of orthogonal polynomials) $\mathbf{y} \in \Gamma$, construct an approximation $u_{h}(T, \cdot, \mathbf{y}) \in H_{h}(D)$ and $p_{h}(T, \cdot, \mathbf{y}) \in L_{h}(D)$ satisfying

$$
\begin{aligned}
&\left(\partial_{t} u_{h}(\mathbf{y}), v_{h}\right)+ a\left(u_{h}(\mathbf{y}), v_{h}\right)+c\left(u_{h}(\mathbf{y}) ; u_{h}(\mathbf{y}), v_{h}\right) \\
&+b\left(v_{h}, p_{h}(\mathbf{y})\right)=\left(f(\mathbf{y}), v_{h}\right), \text { for all } v_{h} \in H_{h}(D), \\
& b\left(u_{h}(\mathbf{y}), q_{h}\right)=0, \text { for all } q_{h} \in L_{h}(D) .
\end{aligned}
$$

Next, we collocate (2.7) on those points and build the discrete solutions $u_{h, p} \in$ $H_{h}(D) \otimes \mathcal{P}_{p}(\Gamma)$ and $p_{h, p} \in L_{h}(D) \otimes \mathcal{P}_{p}(\Gamma)$ by interpolating in $\mathbf{y}$ the collocated solutions, i.e.,

$$
\begin{aligned}
u_{h, p}(T, x, \mathbf{y}) & =\mathcal{I}_{p} u_{h}(T, x, \mathbf{y}) \\
& =\sum_{j_{1}=1}^{p_{1}+1} \cdots \sum_{j_{d}=1}^{p_{d}+1} u_{h}\left(T, x, y_{j_{1}}, \cdots, y_{j_{d}}\right)\left(l_{j_{1}} \otimes \cdots \otimes l_{j_{d}}\right),
\end{aligned}
$$

where, for example, the functions $\left\{l_{j_{k}}\right\}_{k=1}^{d}$ can be taken as Lagrange polynomials. Obviously, the above product requires $\prod_{n=1}^{d}\left(p_{n}+1\right)$ function evaluations.

Because the random input data depend on a finite number of independent random variables and we collocate the weak formulation (2.7) at the zeros of orthogonal polynomials, the solution $u_{h, p}$ becomes a solution of uncoupled deterministic problems as in a Monte Carlo simulation but with much fewer collocation points. 
3. Error analysis for fully discrete schemes. In this section, we carry out an error analysis for the time stepping scheme (BECE) for the approximation of the stochastic Navier-Stokes equation (2.1) in 3 dimensions. The 2-dimensional case should follow similarly. Let $N \in \mathbb{N}_{+}$and consider the uniform partition of the time interval $[0, T]$

$$
0=t_{0}<t_{1}<\cdots<t_{N}=T
$$

with $t_{j}=t_{0}+j \Delta t, j=0,1, \ldots, N$, and the time step $\Delta t=T / N$. For discretizing system (2.7), we apply and study the convergence of the first order backward Euler scheme with a semi-implicit treatment for the nonlinear term

Algorithm 3.1. Given $j \in\{0, \ldots, N-1\}$ and $u_{h}^{j} \in H_{h}, p_{h}^{j} \in L_{h}$, find $u_{h}^{j+1} \in$ $H_{h}, p_{h}^{j+1} \in L_{h}$ satisfying

$$
\begin{gathered}
\left(\frac{u_{h}^{j+1}-u_{h}^{j}}{\Delta t}, v_{h}\right)+a\left(u_{h}^{j+1}, v_{h}\right)+c\left(u_{h}^{j} ; u_{h}^{j+1}, v_{h}\right)+b\left(v_{h}, p_{h}^{j+1}\right) \\
=\left(f^{j+1}, v_{h}\right), \text { for all } v_{h} \in H_{h}, \\
b\left(u_{h}^{j+1}, q_{h}\right)=0, \text { for all } q_{h} \in L_{h} .
\end{gathered}
$$

We will investigate the analyticity of the solution $u_{h}$ with respect to $\mathbf{y}$, which is the core to establish the interpolation error estimates. Introducing the weight function $\sigma(\mathbf{y})=\prod_{n=1}^{d} \sigma_{n}\left(y_{n}\right) \leq 1$, where

$$
\begin{aligned}
& \sigma_{n}\left(y_{n}\right)=1 \text { if } \Gamma_{n} \text { is bounded, } \\
& \sigma_{n}\left(y_{n}\right)=e^{-\lambda_{n}\left|y_{n}\right|} \text { for some } \lambda_{n}>0 \text { if } \Gamma_{n} \text { is unbounded, }
\end{aligned}
$$

and the function space

$$
C_{\sigma}^{0}(\Gamma ; V) \equiv\left\{v: \Gamma \rightarrow V, v \text { continuous in } \mathbf{y}, \max _{\mathbf{y} \in \Gamma}\|\sigma(\mathbf{y}) v(\mathbf{y})\|_{V}<+\infty\right\},
$$

We denote

$$
\Gamma_{n}^{*}=\prod_{\substack{j=1 \\ j \neq n}}^{d} \Gamma_{j} \quad \text { and } \quad \sigma_{n}^{*}=\prod_{\substack{j=1 \\ j \neq n}}^{d} \sigma_{j}
$$

with $y_{n}^{*}$ being an arbitrary element of $\Gamma_{n}^{*}$. Similar to [ZG12], we make the following assumption on $\nu$ and $f$.

Assumption 3.1. In what follows we assume that

- $f \in C_{\sigma}^{0}\left(\Gamma ; L^{2}\left(0, T ; L^{2}(D)\right)\right)$,

- $\nu$ is uniformly bounded away from zero,

- There exists $\gamma_{n}<+\infty$ such that

$$
\left\|\frac{\partial_{y_{n}}^{\ell} \nu(\mathbf{y})}{\nu(\mathbf{y})}\right\|_{L^{\infty}(D)} \leq \gamma_{n}^{\ell} \ell !, \quad \frac{\left[\Delta t \sum_{j=0}^{N-1}\left\|\partial_{y_{n}}^{\ell} f^{j+1}(\mathbf{y})\right\|^{2}\right]^{1 / 2}}{1+\|f(\mathbf{y})\|_{L^{2}\left(0, T ; L^{2}(D)\right)}} \leq \gamma_{n}^{\ell} \ell !
$$

for every $\mathbf{y} \in \Gamma, 1 \leq n \leq d$. 
In the next theorem, we prove that for scheme (BECE), $u_{h}$ is analytic in $\mathbf{y}$; however, its analytical radius may shrink exponentially with the number of time steps.

TheOREM 3.1. Under Assumption 3.1, if the solution $u_{h}^{J}\left(y_{n}, \mathbf{y}_{n}^{*}, x\right)$ to (BECE), as a function of $y_{n}$, satisfies $u_{h}^{J}: \Gamma_{n} \rightarrow C_{\sigma_{n}^{*}}^{0}\left(\Gamma_{n}^{*} ; H_{h}(D)\right)$, then for all $n, 1 \leq n \leq d$, there exists $\alpha_{n}>0$ such that for every $J \in\{1, \ldots, N\}, u_{h}^{J}\left(y_{n}, \mathbf{y}_{n}^{*}, x\right)$ admits an analytic extension $u_{h}^{J}\left(z, \mathbf{y}_{n}^{*}, x\right)$ in the region of the complex plane

$$
\Sigma\left(\Gamma_{n} ; \tau_{n, J}\right) \equiv\left\{z \in \mathbb{C} \mid \operatorname{dist}\left(z, \Gamma_{n}\right) \leq \tau_{n, J}\right\},
$$

with $0<\tau_{n, J}<1 / \alpha_{n}^{J}$.

Proof. At every point $y \in \Gamma$, the $\ell$-th derivative of $u_{h}$ w.r.t. $y_{n}$ satisfies

$$
\begin{aligned}
& \left(\frac{1}{\Delta t} \partial_{y_{n}}^{\ell} u_{h}^{j+1}(\mathbf{y})-\frac{1}{\Delta t} \partial_{y_{n}}^{\ell} u_{h}^{j}(\mathbf{y}), v_{h}\right)+\left(\partial_{y_{n}}^{\ell}\left(\nu(\mathbf{y}) \nabla u_{h}^{j+1}(\mathbf{y})\right), \nabla v_{h}\right) \\
& +\left(\partial_{y_{n}}^{\ell}\left(u_{h}^{j}(\mathbf{y}) \cdot \nabla u_{h}^{j+1}(\mathbf{y})\right), v_{h}\right)=\left(\partial_{y_{n}}^{\ell} f^{j+1}(\mathbf{y}), v_{h}\right), \quad \forall v_{h} \in V_{h}(D),
\end{aligned}
$$

or equivalently

$$
\begin{gathered}
\frac{1}{\Delta t}\left(\partial_{y_{n}}^{\ell} u_{h}^{j+1}(\mathbf{y}), v_{h}\right)-\frac{1}{\Delta t}\left(\partial_{y_{n}}^{\ell} u_{h}^{j}(\mathbf{y}), v_{h}\right)+\sum_{m=0}^{\ell}\left(\begin{array}{c}
\ell \\
m
\end{array}\right)\left(\partial_{y_{n}}^{m} \nu(\mathbf{y}) \nabla \partial_{y_{n}}^{\ell-m} u_{h}^{j+1}(\mathbf{y}), \nabla v_{h}\right) \\
+\sum_{m=0}^{\ell}\left(\begin{array}{c}
\ell \\
m
\end{array}\right)\left(\partial_{y_{n}}^{m} u_{h}^{j}(\mathbf{y}) \cdot \nabla \partial_{y_{n}}^{\ell-m} u_{h}^{j+1}(\mathbf{y}), v_{h}\right)=\left(\partial_{y_{n}}^{\ell} f^{j+1}(\mathbf{y}), v_{h}\right) .
\end{gathered}
$$

This implies that

$$
\begin{gathered}
\left(\nu(\mathbf{y}) \nabla \partial_{y_{n}}^{\ell} u_{h}^{j+1}(\mathbf{y}), \nabla v_{h}\right)+\left(u_{h}^{j}(\mathbf{y}) \cdot \nabla \partial_{y_{n}}^{\ell} u_{h}^{j+1}(\mathbf{y}), v_{h}\right) \\
+\frac{1}{\Delta t}\left(\partial_{y_{n}}^{\ell} u_{h}^{j+1}(\mathbf{y}), v_{h}\right)-\frac{1}{\Delta t}\left(\partial_{y_{n}}^{\ell} u_{h}^{j}(\mathbf{y}), v_{h}\right)=\left(\partial_{y_{n}}^{\ell} f^{j+1}(\mathbf{y}), v_{h}\right) \\
-\sum_{m=1}^{\ell}\left(\begin{array}{c}
\ell \\
m
\end{array}\right)\left[\left(\partial_{y_{n}}^{m} \nu(\mathbf{y}) \nabla \partial_{y_{n}}^{\ell-m} u_{h}^{j+1}(\mathbf{y}), \nabla v_{h}\right)+\left(\partial_{y_{n}}^{m} u_{h}^{j}(\mathbf{y}) \cdot \nabla \partial_{y_{n}}^{\ell-m} u_{h}^{j+1}(\mathbf{y}), v_{h}\right)\right]
\end{gathered}
$$

for all $v_{h} \in V_{h}(D)$. Choosing $v_{h}:=\partial_{y_{n}}^{\ell} u_{h}^{j+1}(\mathbf{y})$ and utilizing the divergence free condition and the skew adjoint properties of the nonlinear term we obtain

$$
\begin{aligned}
& \left\|\sqrt{\nu(\mathbf{y})} \nabla \partial_{y_{n}}^{\ell} u_{h}^{j+1}(\mathbf{y})\right\|^{2}+\frac{1}{2 \Delta t}\left\|\partial_{y_{n}}^{\ell} u_{h}^{j+1}(\mathbf{y})\right\|^{2}-\frac{1}{2 \Delta t}\left\|\partial_{y_{n}}^{\ell} u_{h}^{j}(\mathbf{y})\right\|^{2} \\
& +\frac{1}{2 \Delta t}\left\|\partial_{y_{n}}^{\ell} u_{h}^{j+1}(\mathbf{y})-\partial_{y_{n}}^{\ell} u_{h}^{j}(\mathbf{y})\right\|^{2} \\
& =\left(\partial_{y_{n}}^{\ell} f^{j+1}(\mathbf{y}), \partial_{y_{n}}^{\ell} u_{h}^{j+1}(\mathbf{y})\right)-\sum_{m=1}^{\ell}\left(\begin{array}{c}
\ell \\
m
\end{array}\right)\left[\left(\partial_{y_{n}}^{m} u_{h}^{j}(\mathbf{y}) \cdot \nabla \partial_{y_{n}}^{\ell-m} u_{h}^{j+1}(\mathbf{y}), \partial_{y_{n}}^{\ell} u_{h}^{j+1}(\mathbf{y})\right)\right. \\
& \left.+\left(\frac{\partial_{y_{n}}^{m} \nu(\mathbf{y})}{\nu(\mathbf{y})} \sqrt{\nu(\mathbf{y})} \nabla \partial_{y_{n}}^{\ell-m} u_{h}^{j+1}(\mathbf{y}), \sqrt{\nu(\mathbf{y})} \nabla \partial_{y_{n}}^{\ell} u_{h}^{j+1}(\mathbf{y})\right)\right] \\
& \leq \frac{C_{P}}{\sqrt{\nu_{\min }}}\left\|\partial_{y_{n}}^{\ell} f^{j+1}(\mathbf{y})\right\|\left\|\sqrt{\nu(\mathbf{y})} \nabla \partial_{y_{n}}^{\ell} u_{h}^{j+1}(\mathbf{y})\right\| \\
& +\sum_{m=1}^{\ell}\left(\begin{array}{c}
\ell \\
m
\end{array}\right)\left\|\frac{\partial_{y_{n}}^{m} \nu(\mathbf{y})}{\nu(\mathbf{y})}\right\|_{L^{\infty}(D)}\left\|\sqrt{\nu(\mathbf{y})} \nabla \partial_{y_{n}}^{\ell-m} u_{h}^{j+1}(\mathbf{y})\right\|\left\|\sqrt{\nu(\mathbf{y})} \nabla \partial_{y_{n}}^{\ell} u_{h}^{j+1}(\mathbf{y})\right\|
\end{aligned}
$$




$$
\begin{aligned}
& +\frac{16}{27 \nu_{\min }^{7 / 8}} \sum_{m=1}^{\ell}\left(\begin{array}{c}
\ell \\
m
\end{array}\right)\left\|\partial_{y_{n}}^{m} u_{h}^{j}(\mathbf{y})\right\|^{1 / 4}\left\|\nabla \partial_{y_{n}}^{m} u_{h}^{j}(\mathbf{y})\right\|^{3 / 4}\left\|\sqrt{\nu(\mathbf{y})} \nabla \partial_{y_{n}}^{\ell} u_{h}^{j+1}(\mathbf{y})\right\| \times \\
& \quad \times\left\|\partial_{y_{n}}^{\ell-m} u_{h}^{j+1}(\mathbf{y})\right\|^{1 / 4}\left\|\sqrt{\nu(\mathbf{y})} \nabla \partial_{y_{n}}^{\ell-m} u_{h}^{j+1}(\mathbf{y})\right\|^{3 / 4} \\
& \leq \frac{C_{P}}{\sqrt{\nu_{\min }}}\left\|\partial_{y_{n}}^{\ell} f^{j+1}(\mathbf{y})\right\|\left\|\sqrt{\nu(\mathbf{y})} \nabla \partial_{y_{n}}^{\ell} u_{h}^{j+1}(\mathbf{y})\right\| \\
& \quad+\sum_{m=1}^{\ell}\left(\begin{array}{c}
\ell \\
m
\end{array}\right)\left\|\frac{\partial_{y_{n}}^{m} \nu(\mathbf{y})}{\nu(\mathbf{y})}\right\|_{L^{\infty}(D)}\left\|\sqrt{\nu(y)} \nabla \partial_{y_{n}}^{\ell-m} u_{h}^{j+1}(\mathbf{y})\right\|\left\|\sqrt{\nu(y)} \nabla \partial_{y_{n}}^{\ell} u_{h}^{j+1}(\mathbf{y})\right\| \\
& +\frac{16}{27} \frac{C_{P}^{1 / 2}}{\nu_{\min }^{3 / 2}} \sum_{m=1}^{\ell}\left(\begin{array}{c}
\ell \\
m
\end{array}\right)\left\|\sqrt{\nu(\mathbf{y})} \nabla \partial_{y_{n}}^{m} u_{h}^{j}(\mathbf{y})\right\|\left\|\sqrt{\nu(\mathbf{y})} \nabla \partial_{y_{n}}^{\ell-m} u_{h}^{j+1}(\mathbf{y})\right\|\left\|\sqrt{\nu(\mathbf{y})} \nabla \partial_{y_{n}}^{\ell} u_{h}^{j+1}(\mathbf{y})\right\| .
\end{aligned}
$$

Denoting

$$
F_{m}^{J}=\left[\Delta t \sum_{j=0}^{J-1}\left\|\partial_{y_{n}}^{m} f^{j+1}(\mathbf{y})\right\|^{2}\right]^{1 / 2} \text { and } R_{m}^{J}=\left[\Delta t \sum_{j=0}^{J-1}\left\|\sqrt{\nu(\mathbf{y})} \nabla \partial_{y_{n}}^{m} u_{h}^{j+1}(\mathbf{y})\right\|^{2}\right]^{1 / 2},
$$

and summing from $j=0$ to $J-1$, it gives

$$
\begin{aligned}
\left\|\partial_{y_{n}}^{\ell} u_{h}^{J}\right\|^{2} & +2\left(R_{\ell}^{J}\right)^{2} \leq \frac{2 C_{P}}{\sqrt{\nu_{\min }}} F_{\ell}^{J} R_{\ell}^{J}+2 \sum_{m=1}^{\ell}\left(\begin{array}{c}
\ell \\
m
\end{array}\right)\left\|\frac{\partial_{y_{n}}^{m} \nu(\mathbf{y})}{\nu(\mathbf{y})}\right\|_{L^{\infty}(D)} R_{\ell-m}^{J} R_{\ell}^{J} \\
& +\frac{32}{27} \frac{C_{P}^{1 / 2}}{\Delta t^{1 / 2} \nu_{\min }^{3 / 2}} \sum_{m=1}^{\ell}\left(\begin{array}{c}
\ell \\
m
\end{array}\right) R_{m}^{J-1} R_{\ell-m}^{J} R_{\ell}^{J}
\end{aligned}
$$

This leads us to

$$
\begin{aligned}
\frac{\left\|\partial_{y_{n}}^{\ell} u_{h}^{J}\right\|^{2}}{2 R_{\ell}^{J}}+R_{\ell}^{J} & \leq \frac{C_{P}}{\sqrt{\nu_{\min }}} F_{\ell}^{J}+\sum_{m=1}^{\ell}\left(\begin{array}{c}
\ell \\
m
\end{array}\right)\left\|\frac{\partial_{y_{n}}^{m} \nu(\mathbf{y})}{\nu(\mathbf{y})}\right\|_{L^{\infty}(D)} R_{\ell-m}^{J} \\
+ & \frac{16}{27} \frac{C_{P}^{1 / 2}}{\Delta t^{1 / 2} \nu_{\min }^{3 / 2}} \sum_{m=1}^{\ell}\left(\begin{array}{c}
\ell \\
m
\end{array}\right) R_{m}^{J-1} R_{\ell-m}^{J}
\end{aligned}
$$

Dividing both sides by $\ell$ ! and denoting $S_{m}^{J}=\frac{R_{m}^{J}}{m !}$ gives

$$
\begin{aligned}
\frac{\left\|\partial_{y_{n}}^{\ell} u_{h}^{J}\right\|^{2}}{2 R_{\ell}^{J} \ell !}+S_{\ell}^{J} \leq \frac{C_{P}}{\sqrt{\nu_{\min }}} \gamma_{n}^{\ell}(1 & +\|f\|)+\sum_{m=1}^{\ell} \gamma_{n}^{m} S_{\ell-m}^{J} \\
& +\frac{16}{27} \frac{C_{P}^{1 / 2}}{\Delta t^{1 / 2} \nu_{\min }^{3 / 2}} \sum_{m=1}^{\ell} S_{m}^{J-1} S_{\ell-m}^{J} .
\end{aligned}
$$

Denoting two parameters independent of $J, \ell$ and $u_{h}$

$$
A=\frac{C_{P}}{\sqrt{\nu_{\min }}}(1+\|f\|), \quad \text { and } \quad B=\frac{16}{27} \frac{C_{P}^{1 / 2}}{\Delta t^{1 / 2} \nu_{\min }^{3 / 2}} .
$$

We will prove that there exists $\alpha_{n}>0$ independent of $J, \ell$ and $u_{h}$ such that

$$
S_{\ell}^{J} \leq \alpha_{n}^{\ell J} \text { for all } 1 \leq \ell<\infty, 1 \leq J \leq N .
$$

First, we consider three specific cases: 
1. $\ell=0$ : Take $v=u_{h}^{j+1}(\mathbf{y})$ in (BECE), we have

$$
\begin{aligned}
\frac{1}{2 \Delta t}\left\|u_{h}^{j+1}(\mathbf{y})\right\|^{2} & -\frac{1}{2 \Delta t}\left\|u_{h}^{j}(\mathbf{y})\right\|^{2}+\left\|\sqrt{\nu(\mathbf{y})} \nabla u_{h}^{j+1}(\mathbf{y})\right\|^{2} \\
& \leq \frac{C_{P}^{2}}{2 \nu_{\min }}\left\|f^{j+1}(\mathbf{y})\right\|^{2}+\frac{1}{2}\left\|\sqrt{\nu(\mathbf{y})} \nabla u_{h}^{j+1}(\mathbf{y})\right\|^{2}
\end{aligned}
$$

Summing from $j=0$ to $J-1$ and multiplying by $2 \Delta t$, there follows

$$
\begin{aligned}
\left\|u_{h}^{J}(\mathbf{y})\right\|^{2}+\Delta t \sum_{j=0}^{J-1} & \left\|\sqrt{\nu(\mathbf{y})} \nabla u_{h}^{j+1}(\mathbf{y})\right\|^{2} \\
& \leq \frac{C_{P}^{2}}{\nu_{\min }} \Delta t \sum_{j=0}^{J-1}\left\|f^{j+1}(\mathbf{y})\right\|^{2}+\left\|u_{h}^{0}\right\|^{2},
\end{aligned}
$$

which gives $S_{0}^{J} \leq \xi_{0}, \forall 1 \leq J \leq N$, where $\xi_{0}=\frac{C_{P}}{\sqrt{\nu_{\min }}}(1+\|f\|)+\left\|u_{h}^{0}\right\|$.

2. $\ell=1$ : From (3.3), we have

$$
S_{1}^{J} \leq A \gamma_{n}+\gamma_{n} S_{0}^{J}+B S_{1}^{J-1} S_{0}^{J} \leq A \gamma_{n}+\gamma_{n} \xi_{0}+B \xi_{0} S_{1}^{J-1} .
$$

There follows

$$
S_{1}^{J} \leq\left(A \gamma_{n}+\gamma_{n} \xi_{0}\right) \sum_{j=0}^{J-1}\left(B \xi_{0}\right)^{j} \leq \alpha_{n}^{J}, \forall J \leq N,
$$

with $\alpha_{n}=\max \left\{B \xi_{0}, \frac{B \xi_{0}}{B \xi_{0}-1}\left(A \gamma_{n}+\gamma_{n} \xi_{0}\right)\right\}$.

3. $J=1$ : From (3.3), we have

$$
S_{\ell}^{1} \leq A \gamma_{n}^{\ell}+\sum_{m=1}^{\ell} \gamma_{n}^{m} S_{\ell-m}^{1}=A \gamma_{n}^{\ell}+\xi_{0} \gamma_{n}^{\ell}+\sum_{m=1}^{\ell-1} \gamma_{n}^{m} S_{\ell-m}^{1}
$$

By induction, we will prove that $S_{\ell}^{1} \leq \alpha_{n}^{\ell}, \forall \ell \geq 1, \ell<\infty$. Assuming in addition $\alpha_{n} \geq \max \left\{4 \gamma_{n}, 2\left(A+\xi_{0}\right) \gamma_{n}\right\}$, it gives

$$
\begin{aligned}
S_{\ell}^{1} & \leq \min \left\{\frac{A+\xi_{0}}{2^{\ell}\left(A+\xi_{0}\right)^{\ell}}, \frac{A+\xi_{0}}{4^{\ell}}\right\} \alpha_{n}^{\ell}+\sum_{m=1}^{\ell-1} \frac{\alpha_{n}^{m}}{4^{m}} \alpha_{n}^{\ell-m} \\
& \leq \frac{1}{2} \alpha_{n}^{\ell}+\alpha_{n}^{\ell} \sum_{m=1}^{\ell-1} \frac{1}{4^{m}}<\alpha_{n}^{\ell} .
\end{aligned}
$$

Next, suppose that (3.4) occurs for all $J \leq N$ with $\ell \leq L-1$ and all $J \leq M-1$ with $\ell=L(L \geq 2, M \geq 2)$, we will prove that (3.4) also occurs for $J=M, \ell=L$. Indeed, from (3.3),

$$
S_{L}^{M} \leq A \gamma_{n}^{L}+\sum_{m=1}^{L} \gamma_{n}^{m} S_{L-m}^{M}+B \sum_{m=1}^{L} S_{m}^{M-1} S_{L-m}^{M} .
$$


By induction hypothesis, we have

$$
\begin{aligned}
S_{L}^{M} & \leq A \frac{\alpha_{n}^{L}}{4^{L}}+\xi_{0} \frac{\alpha_{n}^{L}}{4^{L}}+\sum_{m=1}^{L-1} \frac{\alpha_{n}^{m}}{4^{m}} \alpha_{n}^{M L-m M}+B \xi_{0} \alpha_{n}^{M L-L}+B \sum_{m=1}^{L-1} \alpha_{n}^{M L-m} \\
& \leq\left(A+\xi_{0}+B \xi_{0}\right) \alpha_{n}^{M L-1}+\alpha_{n}^{M L-1} \sum_{m=1}^{L-1} \frac{1}{4^{m}}+B \alpha_{n}^{M L-1} \frac{\alpha_{n}}{\alpha_{n}-1} \\
& \leq\left(A+\xi_{0}+B \xi_{0}+\frac{1}{3}+2 B\right) \alpha_{n}^{M L-1}\left(\text { assuming in addition that } \alpha_{n} \geq 2\right) \\
& \left.\leq \alpha_{n}^{M L} \text { (assuming in addition that } \alpha_{n} \geq\left(A+\xi_{0}+B \xi_{0}+\frac{1}{3}+2 B\right)\right)
\end{aligned}
$$

and (3.4) is proved completely.

Back to (3.3), it gives

$$
\frac{\left\|\partial_{y_{n}}^{\ell} u_{h}^{J}\right\|^{2}}{2 R_{\ell}^{J} \ell !}+S_{\ell}^{J} \leq A \gamma_{n}^{\ell}+\sum_{m=1}^{\ell} \gamma_{n}^{m} S_{\ell-m}^{J}+B \sum_{m=1}^{\ell} S_{m}^{J-1} S_{\ell-m}^{J}
$$

for all $\ell$. Employing the above estimation, we get

$$
\frac{\left\|\partial_{y_{n}}^{\ell} u_{h}^{J}\right\|^{2}}{2 R_{\ell}^{J} \ell !} \leq \alpha_{n}^{\ell J}, \forall 1 \leq \ell<\infty, 1 \leq J \leq N
$$

and there holds

$$
\left\|\partial_{y_{n}}^{\ell} u_{h}^{J}\right\| \leq \sqrt{2}(\ell !) \alpha_{n}^{\ell J} .
$$

We now define for every $y_{n} \in \Gamma_{n}$ the power series $u_{h}^{J}: \mathbb{C} \rightarrow C^{0}\left(\Gamma_{n}^{*} ; H_{h}(D)\right)$ as

$$
u_{h}^{J}\left(z, \mathbf{y}_{n}^{*}, x\right)=\sum_{\ell=0}^{\infty} \frac{\left(z-y_{n}\right)^{\ell}}{\ell !} \partial_{y_{n}}^{\ell} u_{h}^{J}\left(y_{n}, \mathbf{y}_{n}^{*}, x\right) .
$$

Hence,

$$
\begin{aligned}
& \sigma_{n}\left(y_{n}\right)\left\|u_{h}^{J}(z)\right\|_{C_{\sigma_{n}^{*}}^{0}\left(\Gamma_{n}^{*}, H_{h}(D)\right)} \leq \sum_{\ell=0}^{\infty} \frac{\left|z-y_{n}\right|^{\ell}}{\ell !} \sigma_{n}\left(y_{n}\right)\left\|\partial_{y_{n}}^{\ell} u_{h}^{J}\left(y_{n}\right)\right\|_{C_{\sigma_{n}^{*}}^{0}\left(\Gamma_{n}^{*}, H_{h}(D)\right)} \\
\leq & \left\|u_{h}^{J}\right\|_{C_{\sigma}^{0}\left(\Gamma ; H_{h}(D)\right)} \sum_{\ell=0}^{\infty}\left(\left|z-y_{n}\right| \alpha_{n}^{J}\right)^{\ell} \\
\leq & \left(\left\|\frac{C_{P}}{\sqrt{\nu_{\min }}}+\right\| u_{h}^{0}\|\|_{C_{\sigma}^{0}(\Gamma ; \mathbb{R})}+\left\|\frac{C_{P}}{\sqrt{\nu_{\min }}} f\right\|_{C_{\sigma}^{0}\left(\Gamma ; L^{2}\left(0, T ; L^{2}(D)\right)\right)}\right) \sum_{\ell=0}^{\infty}\left(\left|z-y_{n}\right| \alpha_{n}^{J}\right)^{\ell}
\end{aligned}
$$

(from (3.5)).

The series converges for all $z \in \mathbb{C}$ satisfying $\left|z-y_{n}\right| \leq \tau_{n, J}<1 / \alpha_{n}^{J}$ and the function $u_{h}^{J}$ admits an analytical extension in the region $\Sigma\left(\Gamma_{n}, \tau_{n, J}\right)$. $\square$

With the analyticity result proved in Theorem 3.1, we proceed to estimate the interpolation error $\varepsilon=u_{h}-u_{h, p}$. The proof follows the same procedure as in [BNT07], and thus, is omitted here.

THEOREM 3.2. Under the assumption of Theorem 3.1, suppose that the joint probability density $\rho$ satisfies

$$
\rho(\mathbf{y}) \leq C_{M} e^{-\sum_{n=1}^{d}\left(\delta_{n} y_{n}\right)^{2}} \quad \forall \mathbf{y} \in \Gamma
$$


for some constant $C_{M}>0$ and $\delta_{n}$ strictly positive if $\Gamma_{n}$ is unbounded and zero otherwise. Then, for any integer $J \in\{1, \ldots, N\}$, there exists a positive constant $C$ independent of $h$ and $p$ such that

$$
\left\|u_{h}^{J}-u_{h, p}^{J}\right\|_{L^{2}(D) \otimes L_{\rho}^{2}(\Gamma)} \leq C \sum_{n=1}^{d} \beta_{n}\left(p_{n}\right) \exp \left(-r_{n, J} p_{n}^{\theta_{n}}\right),
$$

where

$$
\theta_{n}=\beta_{n}=1 \text { and } r_{n, J}=\log \left[\frac{2 \tau_{n, J}}{\left|\Gamma_{n}\right|}\left(1+\sqrt{1+\frac{\left|\Gamma_{n}\right|^{2}}{4 \tau_{n, J}^{2}}}\right)\right] \text { if } \Gamma_{n} \text { is bounded }
$$

and

$$
\theta_{n}=\frac{1}{2}, \beta_{n}=O\left(\sqrt{p_{n}}\right) \text { and } r_{n, J}=\tau_{n, J} \delta_{n} \text { if } \Gamma_{n} \text { is unbounded, }
$$

where $\tau_{n, J}$ is the minimum distance between $\Gamma_{n}$ and the nearest singularity in the complex plane, as defined in Theorem 3.1.

REMARK 3.1. Theorem 3.2 implies that:

- At a fixed time step $J$, the convergence rate of SCMs for bounded random variables is $O(\exp (-p))$ and for unbounded random variables is $O(\exp (-\sqrt{p}))$.

- $\tau_{n, J}$ converges to 0 as $J$ increases, as indicated in Theorem 3.1, and so does $r_{n, J}$. Thus, at a fixed polynomial order $p$, the interpolation error $u_{h}^{J}-u_{h, p}^{J}$ become $O(1)$ as $J \rightarrow \infty$.

4. Numerical examples. To illustrate our numerical analysis, in this section, we present a computational experiment of two-dimensional flow around a circular cylinder with uncertain viscosity, based on the well-known benchmark problem from Shäfer and Turek [ST96]. The exponential decay of interpolation errors and the decrease of $p$-convergence rate in long term have already been shown for various flow experiments. The results we give herein are agree to those in [WK06, FWK08, Loe10]. Our test is programmed using the software package FreeFem ++ [Hec12].

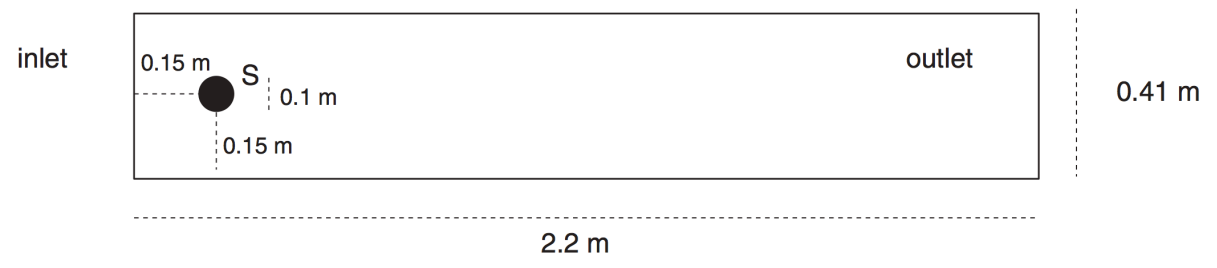

Fig. 4.1: Domain $\Omega$ of the numerical test, from [John04].

Let $\Omega$ be the channel with the cylinder presented in Figure 4.1. We consider the time-dependent incompressible Navier-Stokes equation (2.1) subject to the following random viscosity

$$
\nu=\nu_{0}(1+Y / 10)
$$

where $\nu_{0}=0.8 \times 10^{-3}$ and $Y$ is a uniform random variable of zero mean and unit variance. The cylinder, top and bottom of the channel are prescribed no-slip boundary 
conditions, and the inflow and outflow profiles are

$$
\begin{aligned}
& u_{1}(0, y)=u_{1}(2.2, y)=\frac{6}{0.41^{2}} y(0.41-y), \\
& u_{2}(0, y)=u_{2}(2.2, y)=0 .
\end{aligned}
$$

Due to the randomness of the viscosity, the Reynolds number considered in this test is random. Based on the inflow velocity and the diameter of the cylinder $L=0.1$, it satisfies $112.5 \leq R e \leq 137.5$. For this range of Reynolds number, the flow is in the laminar regime with a Kármán vortex street developing behind the cylinder. This results in a periodic response of the lift and drag coefficients. In what follows, we will investigate the mean and error evolution of these two quantities simulated by SCM. By $C_{h, p}^{l \mid d}$, we denote the mean lift/drag coefficients corresponding to the fully discrete solutions in physical and probability spaces. In order to estimate the error, we compute a very high resolution approximate solution using SCM of $20^{\text {th }}$-order and suppose it to be the true solution in probability space, the lift/drag coefficients corresponding to which are denoted by $C_{h}^{l \mid d}$.

The experiment is carried out up to $T=50$, with zero forcing term and initial condition. The solutions are computed with Taylor-Hood elements on a triangular mesh providing 69174 total DOFs, refined near the cylinder, and time step $\Delta t=0.005$. At each collocation point of $Y$, we employ the Crank-Nicolson scheme to solve for the Navier-Stokes solutions. The scheme reads:

Given $j \in\{0, \ldots, N-1\}$ and $u_{h}^{j} \in H_{h}, p_{h}^{j} \in L_{h}$, find $u_{h}^{j+1} \in H_{h}, p_{h}^{j+1} \in L_{h}$ satisfying

$$
\begin{gathered}
\left(\frac{u_{h}^{j+1}-u_{h}^{j}}{\Delta t}, v_{h}\right)+\frac{1}{2}\left(a\left(u_{h}^{j+1}, v_{h}\right)+a\left(u_{h}^{j}, v_{h}\right)\right)+b\left(v_{h}, p_{h}^{j+1}\right) \\
+\frac{1}{2}\left(c\left(u_{h}^{j+1} ; u_{h}^{j+1}, v_{h}\right)+c\left(u_{h}^{j} ; u_{h}^{j}, v_{h}\right)\right)=\left(f^{j+1 / 2}, v_{h}\right), \text { for all } v_{h} \in H_{h}, \\
b\left(u_{h}^{j+1}, q_{h}\right)=0, \text { for all } q_{h} \in L_{h} .
\end{gathered}
$$

Fixed point iterations are applied to solve the nonlinear system with a $\| u_{(i+1)}-$ $u_{(i)} \|_{H_{1}(\Omega)}<10^{-10}$ as a stopping criterion. The numerical methods and space-time resolution we use herein were verified in deterministic problem to give a good approximation of lift and drag coefficients, [John04].

Figure 4.2 show the instantaneous mean of lift and drag coefficients given by SCM. It can be seen that both the mean $C_{h, p}^{l}$ and $C_{h, p}^{d}$ oscillate periodically around a constant value. After a short time agreeing with the reference solution, SCM starts to break down. The higher order the method is, the later the divergence occurs.

The evolution of errors of SCM at polynomial orders $p=1,2,3,4,6$ are shown in Figure 4.3. We observe that for all $p$ plotted, the interpolation errors become $O(1)$ in long term. This confirms our claim in Remark 3.1. Finally, we plot the convergence of SCM at different time level in Figure 4.4. The method exhibits good convergence rate in short term $(t=2)$. At $t=20$, the error grows significantly, but still decays in $p$. At $t=50$, the error is almost steady: increasing the polynomial order up to 6 does little help. 

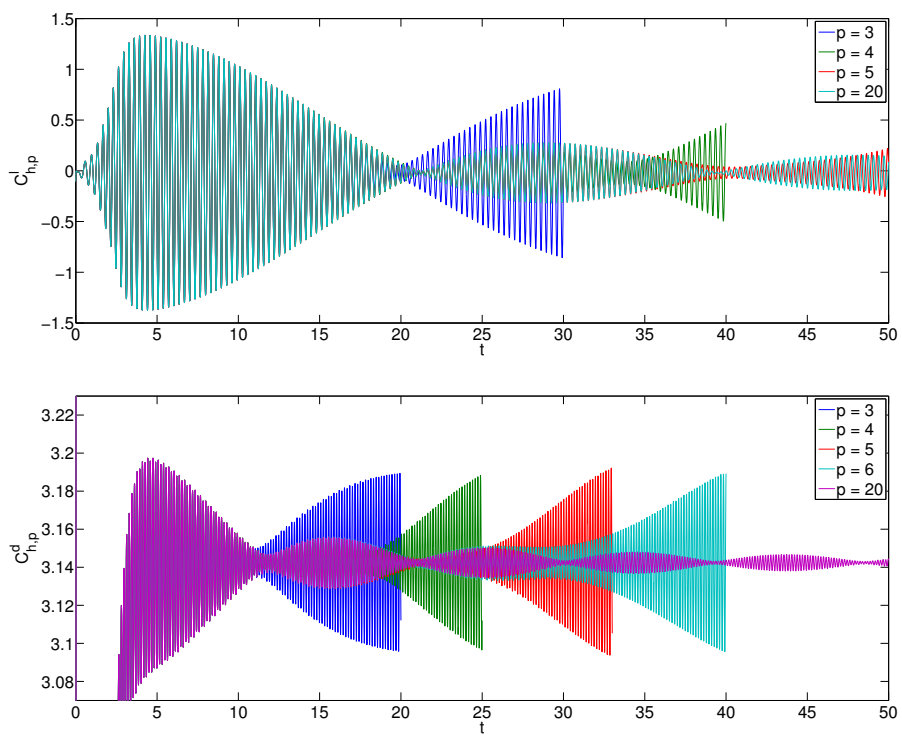

Fig. 4.2: Evolution of mean of lift (upper) and drag (lower) coefficient.
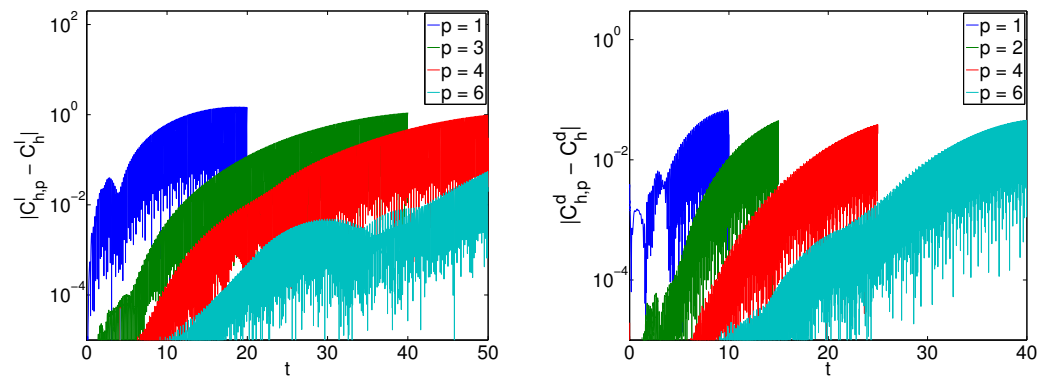

Fig. 4.3: Evolution of error of lift (left) and drag (right) coefficient.
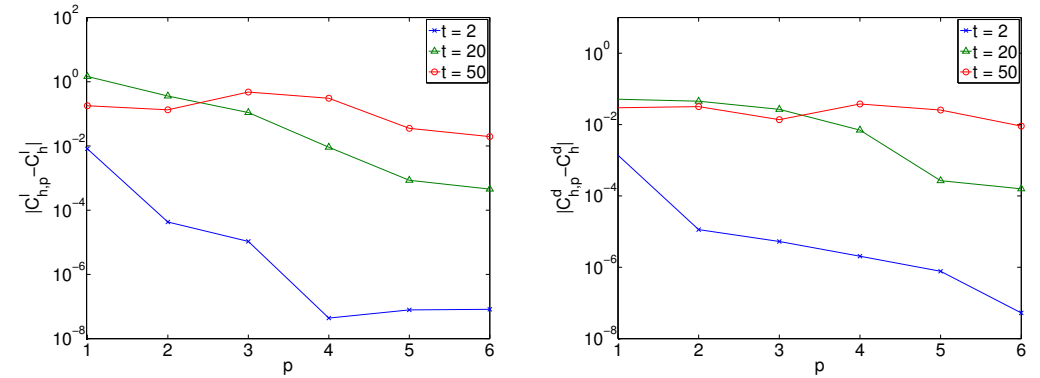

Fig. 4.4: $p$-Convergence rate of SCM for the simulation of flow around a cylinder at different time level. 
5. Conclusions. In this paper, an error analysis of stochastic collocation methods for a fully discrete Navier-Stokes scheme with random input data was carried out. At each fixed time level, we proved the exponential convergence in the probability space. At each fixed total number of collocation points, our analysis indicated that the interpolation error may grow to $O(1)$ in long term. A numerical example of $2 \mathrm{D}$ flow around a cylinder is given to illustrate our theoretical results.

The analysis provided herein deals with the first order (BECE) scheme, to help us focus on interpolation error and keep things simple. Extension to higher order, more applicable semi-implicit and fully implicit Navier-Stokes schemes is worth further study.

\section{REFERENCES}

[ACH06] G. Apte, S. Constantinescu, F. Ham, G. Iaccarino, P. Mahesh, And K. Moin, Large-eddy simulation of reacting turbulent flows in complex geometries, J. Appl. Mech., 73:364-371, 2006.

[BDK82] G.A. BAKER, V. Dougalis, AND O. KARAKASHIAn, On a higher order accurate, fully discrete galerkin approximation to the Navier-Stokes equations, Math. Comp., 39:339375, 1982

[BNT07] I. Babuska, F. Nobile, And R. Tempone, A Stochastic Collocation Method for Elliptic Partial Differential Equations with Random Input Data, SIAM Journal on Numerical Analysis, 45 (2007), pp. 1005-1034.

[BS08] S. Brenner And R. Scott, The Mathematical Theory of Finite Element Methods, Springer, 3rd edition, 2008.

[DFJ74] T. Dupont, G. Fairweather And J. P. Johnson, Three-level Galerkin methods for parabolic equations, SIAM J. Numer. Anal., v.11, 1974, pp. 392-410.

[FWK08] J. FOO, X. WAN, AND G. KARNIADAKIS, The multi-element probabilistic collocation method (ME-PCM): Error analysis and applications, Journal of Computational Physics, 227 (2008), pp. 9572-9595.

[G89] M.D. GunzBurger, Finite Element Methods for Viscous Incompressible Flows - A Guide to Theory, Practices, and Algorithms, Academic Press, 1989.

[GR79] V. Girault And P.A. Raviart, Finite Element Approximations of the Navier-Stokes Equations, Lecture Notes in Mathematics. Springer-Verlag, New York, 1979.

[GWZ13] M. Gunzburger, C. Webster, G. Zhang, An Adaptive Wavelet Stochastic Collocation Method for Irregular Solutions of Partial Differential Equations with Random Input Data, Springer Lecture Notes on Computational Science, to appear.

[Hec12] F. Hecht, New development in freefem ++ , J. Numer. Math. 20 (2012), no. 3-4, 251-265.

[HR90] J.G HEYWOOD AND R. RANNACHER, Finite element approximation of the nonstationary Navier-Stokes problem, IV. Error analysis for second-order time discretization, SIAM J. Numer. Anal., 27(2):353-384, 1990.

[HS07] Y. HE AND W. Sun, Stability and convergence of the Crank-Nicolson/Adams-Bashforth scheme for the time-dependent Navier-Stokes equations, SIAM J. Numer. Anal., 45:837-869, Feb. 2007.

[Ing13] R. INGRAM, Unconditional convergence of high-order extrapolations of the CrankNicolson, finite element methods for the Navier-Stokes equations, IJNAM, 10(2), pp. 257-297, 2013.

[John04] V. John, Reference values for drag and lift of a two-dimensional time-dependent flow around a cylinder, Int. J. Numer. Meth. Fluids 44 (2004) 777-788.

[Lay08] W. LAYTON, Introduction to the Numerical Analysis of Incompressible Viscous Flows, Computational Science and Engineering Series, SIAM, 2008.

[Loe87] M. Loغ̀ve, Probability Theory I, Grad. Texts in Math. 45, Springer-Verlag, New York, 1987.

[Loe10] G. Loeven, Efficient uncertainty quantification in computational fluid dynamics, Ph.D. thesis, TU Delft, 2010.

[Luc04] D. LuCor, Generalized Polynomial Chaos: Applications to Random Oscillators and Flow-structure Interactions, Ph.D. thesis, Brown University, Division of Applied Mathematics, 2004.

[MHZ05] L. Mathelin, M. Hussaini, And T. ZANG, Stochastic approaches to uncertainty quantification in CFD simulations, Numer. Algorithms, 38 (2005), pp. 209-236. 
[MKNG04] O. P. Le Maître, O. M. Knio, H. N. Najm, and R. G. Ghanem, Uncertainty propagation using Wiener-Haar expansions, J. Comput. Phys., 197 (2004), pp. 28-57.

[Mmou06] K. Matsuzaki, M. Munekata, H. Ohba, and Ushijima, Numerical study on particle motions in swirling flows in a cyclone separator, J. Therm. Sci., 15(2):181-186, 2006.

[MNGK04] O. P. Le Maître, H. N. Najm, R. G. Ghanem, and O. M. Knio, Multi-resolution analysis of Wiener-type uncertainty propagation schemes, J. Comput. Phys., 197 (2004), pp. 502-531.

[NT09] F. Nobile And R. Tempone, Analysis and implementation issues for the numerical approximation of parabolic equations with random coefficients, Internat. J. Numer. Methods Engrg., 80 (2009), pp. 979-1006.

[NTW08] F. Nobile, R. Tempone, and C. G. Webster, A sparse grid stochastic collocation method for partial differential equations with random input data, SIAM J. Numer. Anal., 46 (2008), pp. 2309-2345.

[NTW08b] F. Nobile, R. Tempone, and C. G. Webster, An anisotropic sparse grid collocation method for elliptic partial differential equations with random input data, SIAM J. Numer. Anal. 46 (5) (2008) 2411-2442.

[SS02] J.N. Sorensen And W.Z Shen, Numerical modeling of wind turbine wakes, J. Fluids Eng., 124:393-399, Jun. 2002.

[ST96] M. ShäFer AND S. TUREK, Benchmark computations of laminar flow around cylinder, Flow Simulation with High-Performance Computers II, Vieweg, 1996.

[TS07] R.A. TODOR, C. SCHWAB, Convergence rates for sparse chaos approximations of elliptic problems with stochastic coefficients, IMA J. Numer. Anal. 27 (2) (2007) 232-261.

[TT00] M. TABATA AND D. TAGAMI, Error estimates for finite element approximations of drag and lift in nonstationary Navier-Stokes flows, Japan J. Indust. Appli. Math., 17:371$389,2000$.

[WK05] X.L. WAN AND G.E. KARNIADAKIS, An adaptive multi-element generalized polynomial chaos method for stochastic differential equations, J. Comput. Phys. 209 (2) (2005) 617-642.

[WK06] X.L. WAN AND G.E. KARNIADAKIS, Long-term behavior of polynomial chaos in stochastic flow simulations, Comput. Methods Appl. Mech. Engrg. 195 (2006) 5582-5596.

[WK06b] X.L. WAN AND G.E. KARNIADAKIS, Multi-element generalized polynomial chaos for arbitrary probability measures, SIAM J. Sci. Comput. 28 (3) (2006) 901-928.

[WLB07] J.A.S. Witteveen, G.J.A. Loeven, And H. BiJl, Quantifying the effect of physical uncertainties in unsteady fluid-structure interaction problems, in Proceedings of the 48th AIAA/ASME/ASCE/AHS/ASC Structures, Structural Dynamics and Materials Conference, AIAA paper 2007-1942, Honolulu (HI), United States.

[WLSB08] J.A.S. Witteveen, G.J.A. Loeven, S. SARKar, And H. BiJl, Probabilistic collocation for period-1 limit cycle oscillations, Journal of Sound and Vibration 311(1-2), 421439.

[XH05] D. XiU and J. S. Hesthaven, High-order collocation methods for differential equations with random inputs, SIAM J. Sci. Comput., 27 (2005), pp. 1118-1139.

[ZG12] G. Zhang AND M. GunZBURGer, Error analysis of a stochastic collocation method for parabolic partial differential equations with random input data, SIAM Journal on Numerical Analysis, 50 (2012), pp. 1922-1940. 